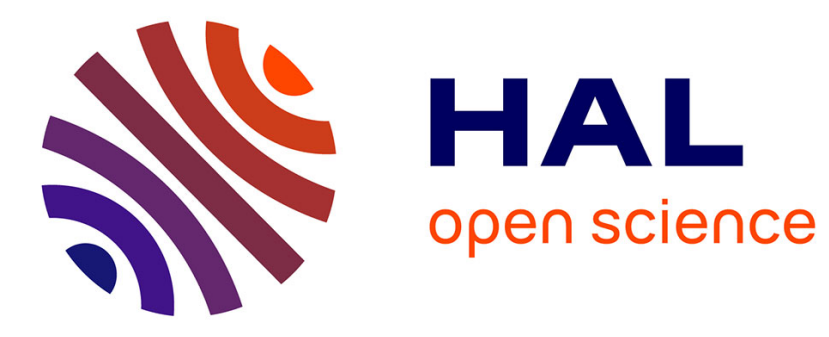

\title{
Exploring the power of converse events
}

\author{
Guillaume Aucher, Andreas Herzig
}

\section{To cite this version:}

Guillaume Aucher, Andreas Herzig. Exploring the power of converse events. Patrick Girard; Olivier Roy; Mathieu Marion. Dynamic formal epistemology, 351, Springer, pp.51-74, 2011, Synthese Library book series (SYLI), 978-94-007-0073-4. 10.1007/978-94-007-0074-1_4 . hal-00557002v2

\section{HAL Id: hal-00557002 \\ https://hal.science/hal-00557002v2}

Submitted on 29 Sep 2011

HAL is a multi-disciplinary open access archive for the deposit and dissemination of scientific research documents, whether they are published or not. The documents may come from teaching and research institutions in France or abroad, or from public or private research centers.
L'archive ouverte pluridisciplinaire HAL, est destinée au dépôt et à la diffusion de documents scientifiques de niveau recherche, publiés ou non, émanant des établissements d'enseignement et de recherche français ou étrangers, des laboratoires publics ou privés. 


\title{
Exploring the power of converse events
}

\author{
Guillaume Aucher and Andreas Herzig
}

$15 / 06 / 09$

\begin{abstract}
Dynamic epistemic logic as viewed by Baltag, Moss and Solecki (BMS) and propositional dynamic logic (PDL) offer different semantics of events. On the one hand, BMS adds dynamics to epistemic logic by introducing so-called event models as syntactic objects into the language. On the other hand, PDL has instead transition relations between possible worlds. This last approach allows to easily introduce converse events. In this paper we add epistemics to this, and call the resulting logic epistemic dynamic logic (EDL). We show that BMS can be translated into EDL thanks to this use of the converse operator: it enables us to translate the structure of the event model directly within a particular axiomatization of EDL, without having to refer to a particular epistemic event model in the language (as done in BMS). It follows that EDL is more expressive and general than BMS and we characterize semantically and syntactically in EDL this embedding of BMS.
\end{abstract}

\section{Introduction}

Aim: reason about perception of events. Accounting for various modes of perception of events is the aim of a family of formal systems called dynamic epistemic logics. These logics add dynamics to Hintikka's epistemic logic via transformations of its models.

The focus of dynamic epistemic logics is typically on epistemic events. The simplest case of epistemic event is public announcement à la Plaza [15]. Another example is group announcement à la Gerbrandy [9]. Note that updates of dynamic epistemic logics differ from Katsuno-Mendelzon-like updates as studied in the AI literature [12] since these updates always involve a factual change in the situation at stake.

In [6, [] ], Baltag, Moss and Solecki proposed a dynamic epistemic logic that was very influential. We refer to it in this paper by the term BMS. It has been shown that their account subsumes all other dynamic epistemic logics. The semantics of BMS is based on two kinds of models: a static model $M^{s}$ (called state model by Baltag, $s$ in $M^{s}$ for $s$ tatic) and a (finite) event model $A$ (called epistemic action model by Baltag). $M^{s}$ models the actual world and the agents' 
beliefs about it, and is nothing but a good old epistemic model à la Hintikka. $A$ models the actual event taking place and the agents' beliefs about it. An agent's beliefs can be uncertain (event $a$ occurred, but agent cannot distinguish occurrence of $a$ from occurrence of $a^{\prime}$ ) and even unsound ( $a$ occurred, but agent wrongly perceived some $a^{\prime}$ ). $M^{s}$ and $A$ are then combined by a restricted product construction which defines the situation after the actual event took place, viz. the resulting actual world, and the agents' beliefs about it.

In this paper, our first aim is to enrich the (dynamic) epistemic language with a modal operator expressing what was true before an event occurred. Our second aim is to propose a unified language which does not refer in its syntax to an event model as in the BMS formalism. Indeed, as its name says, this model is a semantic object. So it seems to us inappropriate to introduce it directly into the syntax of the language (although the way it is actually done in the BMS formalism is formally correct).

Semantics of events: products vs. accessibility relations. Expressing within the BMS formalism what was true before an event $a$ occurred, i.e. giving semantics to the converse event $a^{-}$is not simple partly because the formal definition of what is true after an event $a$ occurs is already rather involved.

On the other hand, in PDL [10], the effects of events are interpreted as transition relations on possible worlds, and not as restricted products of models as in BMS. Converse events $a^{-}$can then easily be interpreted by inverting the accessibility relation associated to $a$. The resulting logic is called the tense extension of PDL. To this we then add an epistemic accessibility relation. We call (tensed) Epistemic Dynamic Logic EDL the combination of epistemic logic and PDL with converse.

A semantics in terms of transition relations is more flexible than the BMS product semantics: we have more options concerning the interaction between events and beliefs. In Section 2 , we will propose an account that captures this relationship more explicitly than the BMS product semantics does by means of constraints on the respective accessibility relations: a no-forgetting and a no-learning constraint, and a constraint of epistemic determinism.

Translating BMS into EDL. To demonstrate the power of our approach we will provide a translation from BMS to EDL. To do so, we will express the structure of an event model $A$ by a nonlogical theory $\Gamma(A)$ of EDL, and prove that any formula $\varphi$ is valid in BMS if and only if it is a logical consequence of $\Gamma(A)$ in EDL. We will also show that $\Gamma(A)$ actually characterizes the EDLmodels which are generated in the 'BMS style' by an event model $A$.

So, unlike BMS, we avoid referring to a semantical structure (i.e. the BMS event model $A$ ) in the very definition of the language. Encoding the structure of a BMS event model $A$ by a nonlogical theory $\Gamma(A)$ of EDL is done thanks

${ }^{1}$ EDL is related to Segerberg's Doxastic Dynamic Logic DDL [19, 20]]. But research on DDL focusses mainly on its relation with AGM theory of belief revision, and studies particular events of the form $+\varphi$ (expansion by $\varphi$ ), $* \varphi$ (revision by $\varphi$ ), and $-\varphi$ (contraction by $\varphi$ ). 
to converse events. For example $[a] B_{j}\left(\left\langle a^{-}\right\rangle \top \vee\left\langle b^{-}\right\rangle \top\right)$ expresses that agent $j$ perceives the occurrence of $a$ as that of either $a$ or $b$.

Organization of the paper. This paper is organized as follows. In section $\square$ we introduce a language of belief, events and converse events. Then we provide a semantics for that language, and define our logic EDL. In section B we give BMS's restricted product semantics for the fragment of the language without converse, and define its logic, also called BMS. In section 4 we provide two embeddings of BMS into EDL: a 'semantic' one and a 'syntactic' one based on a theory $\Gamma(A)$ associated to each event model $A$ (we prove that the consequences of $\Gamma(A)$ in EDL match the BMS-validities). In section $\square$ we compare our formalism with van Benthem and Pacuit's logic ETL and other related work. Finally, we conclude in section $\mathbf{6}$.

\section{EDL: Epistemic Dynamic Logic with converse}

\subsection{The language $\mathcal{L}_{\text {EDL }}$ of EDL}

In this paper, $\Phi$ is a countable set of propositional symbols, $G$ is a finite set of agent symbols, and $E$ is a finite set of event symbols. (Finiteness of $E$ will be crucial for our results, cf. Definition 2.5.)

Definition 2.1 (Language $\mathcal{L}_{\mathrm{EDL}}$ )

The language $\mathcal{L}_{\mathrm{EDL}}$ is defined as follows

$$
\mathcal{L}_{\mathrm{EDL}}: \varphi::=\top|p| \neg \varphi|\varphi \wedge \varphi| B_{j} \varphi|[a] \varphi|\left[a^{-}\right] \varphi,
$$

where $p$ ranges over $\Phi, j$ over $G$ and $a$ over $E$.

The dual modal operators $\langle a\rangle$ and $\left\langle a^{-}\right\rangle$are defined as follows: $\langle a\rangle \varphi$ abbreviates $\neg[a] \neg \varphi ;\left\langle a^{-}\right\rangle \varphi$ abbreviates $\neg\left[a^{-}\right] \neg \varphi$.

We define the language $\mathcal{L}_{\mathrm{BMS}}$ as the sub-language of $\mathcal{L}_{\mathrm{EDL}}$ without converse operators $a^{-}$and the language $\mathcal{L}$ as the sub-language of $\mathcal{L}_{\mathrm{EDL}}$ without dynamic operators $a^{-}$and $a$.

The formula $[a] \varphi$ reads " $\varphi$ will hold after every possible occurrence of event $a^{\prime \prime}$. $\left[a^{-}\right] \varphi$ reads " $\varphi$ held before $a^{\prime \prime}$. So $[a] B_{j}\left[a^{-}\right] \perp$ is an $\mathcal{L}_{\text {EDL }}$-formula that is not in $\mathcal{L}_{\text {BMS }}$.

\subsection{Semantics of EDL}

When designing models of events and beliefs the central issue is to account for the interplay between these two concepts. In our PDL-based semantics this is done by means of constraints on the respective accessibility relations.

Definition 2.2 (EDL-model, no-forgetting, no-learning, epistemic determinism)

An $E D L$-model is a tuple $M=(W, R, \mathcal{R}, V)$ such that 
- $W$ is a non-empty set of possible worlds;

- $R: G \rightarrow 2^{W \times W}$ assigns an accessibility relation to each agent;

- $\mathcal{R}: E \rightarrow 2^{W \times W}$ assigns an accessibility relation to each possible event; and

- $V: \Phi \rightarrow 2^{W}$ is a valuation.

We write $R_{j}$ and $\mathcal{R}_{a}$ instead of $R(j)$ and $\mathcal{R}(a)$, and define $R_{j}(w)=\left\{v \mid w R_{j} v\right\}$ and $\mathcal{R}_{a}^{-1}(v)=\left\{w \mid w \in \mathcal{R}_{a}^{-1}(v)\right\}=\left\{w \mid v \in \mathcal{R}_{a}(w)\right\}$.

Moreover an EDL-model satisfies the constraints of no-forgetting, no-learning and epistemic determinism:

nf If $v^{\prime} \in\left(\mathcal{R}_{a} \circ R_{j}\right)(w)$ then there is $b \in E$ such that $v^{\prime} \in\left(R_{j} \circ \mathcal{R}_{b}\right)(w)$.
nl If $\left(\mathcal{R}_{a} \circ R_{j} \circ \mathcal{R}_{b}^{-1}\right)(w) \neq \emptyset$ then $\left(R_{j} \circ \mathcal{R}_{b}\right)(w) \subseteq\left(\mathcal{R}_{a} \circ R_{j}\right)(w)$.
ed If $v_{1}, v_{2} \in \mathcal{R}_{a}(w)$ then $R_{j}\left(v_{1}\right)=R_{j}\left(v_{2}\right)$.

The no-forgetting principle says that if after an event $a$ agent $j$ considers a world $v^{\prime}$ possible, then before this event $a$ agent $j$ already considered possible

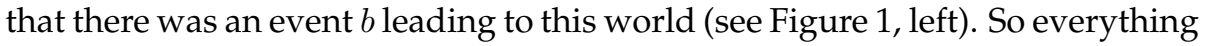
agent $j$ considers possible after the performance of an event stems from what she considered possible before the event. This principle is a generalization of the perfect-recall principle [8].

To understand the no-learning principle, also known as no miracles [24], assume that agent $j$ perceives the occurrence of $a$ as that of $b_{1}, b_{2} \ldots$ or $b_{n}$. Then, informally, the no-learning principle says that all such alternatives resulting from occurrence of $b_{1}, b_{2}, \ldots, b_{n}$ in $j^{\prime}$ s alternatives before $a$ are indeed alternatives after $a$. In a sense there is no miracles: everything the agent was supposed to consider possible after the event is indeed considered possible after the event (if the latter actually takes place). Formally, assume that agent $j$ perceives $b$ as a possible alternative of $a$, i.e. $\left(\mathcal{R}_{a} \circ R_{j} \circ \mathcal{R}_{b}^{-1}\right)(w) \neq \emptyset$. If at $w$ world $v^{\prime}$ was a possible outcome of event $b$ for $j$, then $v^{\prime}$ is possible for $j$ at some $v \in \mathcal{R}_{a}(w)$ (see Figure 开, middle).

Finally, the epistemic determinism principle says that an agent's epistemic state after an event does not depend on the particular nondeterministic outcome. Formally, suppose we have $w \mathcal{R}_{a} v_{1}$ and $w \mathcal{R}_{a} v_{2}$. Then ed forces that the epistemic states at $v_{1}$ and $v_{2}$ are identical: $R_{j}\left(v_{1}\right)=R_{j}\left(v_{2}\right)$ (see Figure $\mathbb{\Pi}$, right).

These three constraints delimit the class of events $E$ we consider. Our events are such that the epistemic state of an agent after the occurrence of an event depends only on the previous epistemic state of the agent and on how the event is perceived by the agent, and not on which facts hold in the world before or after the event. This feature of our events is formally captured by Proposition 2.3 below: $R_{j}(w)$ is the epistemic state of the agent before the 


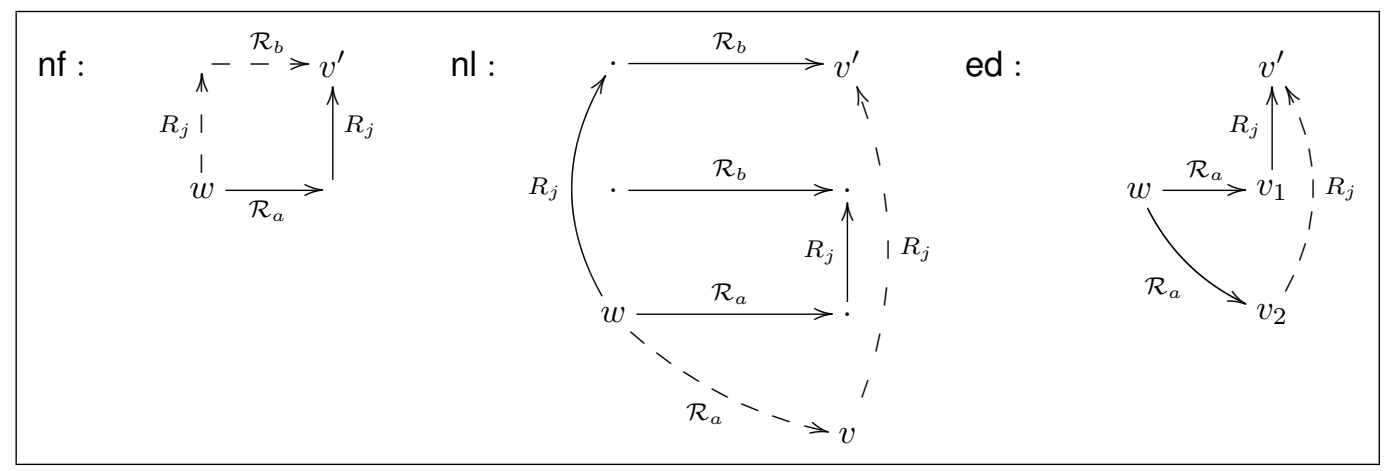

Figure 1: no-forgetting, no-learning and epistemic determinism constraints

event and $A_{a, w}=\left\{b \in E \mid\left(\mathcal{R}_{a} \circ R_{j} \circ \mathcal{R}_{b}^{-1}\right)(w) \neq \emptyset\right\}$ is intuitively the set of events that agent $j$ considers as possibly occurring while event $a$ is in fact occurring at world $w$. For example the event of an agent testing whether $\varphi$ is the case is not an event of the set of atomic events $E$. Indeed the epistemic state of this agent after the test (the agent knowing whether $\varphi$ is true) depends on the actual state of the world (whether $\varphi$ is true or not). In this example the no-learning constraint is violated. Another example of an event which is not dealt with by our formalism is that of tossing a coin and looking at it. In this example, the epistemic state of the agent after the toss depends on the state of the world after the event, i.e. whether the coin lands heads or tails up. Here the epistemic determinism constraint is violated. On the other hand, both public and private announcements are dealt with by our framework. More generally, any kind of announcement (public, private...) about any kind of information (epistemic, stating that an event just occurred...) is dealt with by our framework. Our events are sometimes called ontic events, feedback-free events or uninformative events [11, Z].

Proposition 2.3 Let $M=(W, R, \mathcal{R}, V)$ be a tuple. $M$ is an $E D L$-model, i.e. $M$ satisfies $n f, n l$, ed, iff for all $j \in G$, all $w \in M$, all $a \in E$, all $w^{\prime} \in \mathcal{R}_{a}(w)$,

$$
R_{j}\left(w^{\prime}\right)=\bigcup\left\{\mathcal{R}_{b}(v) \mid b \in A_{a, w}, v \in R_{j}(w)\right\}
$$

where $A_{a, w}=\left\{b \in E \mid \mathcal{R}_{a} \circ R_{j} \circ \mathcal{R}_{b}^{-1}(w) \neq \emptyset\right\}$.

PROOF.

- Assume $M$ satisfies $\mathrm{nf}, \mathrm{nl}$ and ed.

- Let $v^{\prime} \in R_{j}\left(w^{\prime}\right)$. Then $v^{\prime} \in\left(\mathcal{R}_{a} \circ R_{j}\right)(w)$. So by nf there is $b \in E$ and $v \in R_{j}(w)$ such that $v^{\prime} \in \mathcal{R}_{b}(v)$. So $\left(\mathcal{R}_{a} \circ R_{j} \circ \mathcal{R}_{b}^{-1}\right)(w) \neq \emptyset$ and $b \in A_{a, w}$. So $v^{\prime} \in \bigcup\left\{\mathcal{R}_{b}(v) \mid b \in A_{a, w}, v \in R_{j}(w)\right\}$. 
- Let $v^{\prime} \in \bigcup\left\{\mathcal{R}_{b}(v) \mid b \in A_{a, w}, v \in R_{j}(w)\right\}$. Then there is $b \in E$ such that $v^{\prime} \in\left(R_{j} \circ \mathcal{R}_{b}\right)(w)$ and $\left(\mathcal{R}_{a} \circ R_{j} \circ \mathcal{R}_{b}^{-1}\right)(w) \neq \emptyset$. So by $\mathrm{nl}$, $v^{\prime} \in\left(\mathcal{R}_{a} \circ R_{j}\right)(w)$, i.e. there is $w^{\prime \prime} \in \mathcal{R}_{a}(w)$ such that $v^{\prime} \in R_{j}\left(w^{\prime \prime}\right)$. Then by ed, $v^{\prime} \in R_{j}\left(w^{\prime}\right)$.

- Assume $M$ satisfies $\left({ }^{*}\right)$.

nf Assume that $v^{\prime} \in\left(\mathcal{R}_{a} \circ R_{j}\right)(w)$. Then there is $w^{\prime} \in \mathcal{R}_{a}(w)$ such that $v^{\prime} \in R_{j}\left(w^{\prime}\right)$. By $\left(^{*}\right)$ there is $b \in A_{a, w}$ and $v \in R_{j}(w)$ such that $v^{\prime} \in$ $\mathcal{R}_{b}(v)$. So there is $b \in E$ such that $v^{\prime} \in R_{j} \circ \mathcal{R}_{b}(w)$.

$\mathrm{nl}$ Assume that $\left(\mathcal{R}_{a} \circ R_{j} \circ \mathcal{R}_{b}^{-1}\right)(w) \neq \emptyset$ and $v^{\prime} \in\left(R_{j} \circ \mathcal{R}_{b}\right)(w)$. Then there is $v \in R_{j}(w)$ and $b \in A_{a, w}$ such that $v^{\prime} \in \mathcal{R}_{b}(v)$. So $v^{\prime} \in R_{j}\left(w^{\prime}\right)$ for all $w^{\prime} \in \mathcal{R}_{a}(w)$, i.e. $v^{\prime} \in\left(\mathcal{R}_{a} \circ R_{j}\right)(w)$.

ed is clearly fulfilled.

QED

\section{Definition 2.4 (Truth conditions for $\mathcal{L}_{\text {EDL }}$ )}

The semantics of $\mathcal{L}_{\text {EDL }}$ is defined inductively as follows. Let $M$ be an EDLmodel and $w \in M$.

$$
\begin{array}{lll}
M, w \models \top & & \\
M, w \models p & \text { iff } & w \in V(p) \\
M, w \models \varphi \wedge \varphi^{\prime} & \text { iff } & M, w \models \varphi \text { and } M, w=\varphi^{\prime} \\
M, w \models B_{j} \varphi & \text { iff } & \text { for all } v \in R_{j}(w), M, v \models \varphi \\
M, w \models[a] \varphi & \text { iff } & \text { for all } v \in \mathcal{R}_{a}(w), M, v \models \varphi \\
M, w \models\left[a^{-}\right] \varphi & \text { iff } & \text { for all } v \in \mathcal{R}_{a}^{-1}(w), M, v \models \varphi .
\end{array}
$$

Truth of $\varphi$ in a EDL-model $M$ is written $M \models \varphi$ and is defined as: $M, w=\varphi$

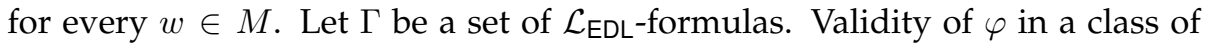
EDL-models $\mathcal{M}$ is written $\mathcal{M} \models \varphi$ and is defined as $M \models \varphi$ for all $M \in \mathcal{M}$. The (global) consequence relation is defined by:

$\Gamma \models_{\text {EDL }} \varphi$ iff for every EDL-model $M$, if $M \models \psi$ for every $\psi \in \Gamma$ then $M \models \varphi$.

For example we have

and

$$
\left\{[b] \varphi,\langle a\rangle B_{j}\left\langle b^{-}\right\rangle \top\right\} \models \operatorname{EDL}[a] B_{j} \varphi
$$

$$
\models \mathrm{EDL}\left(B_{j}[b] \varphi \wedge\langle a\rangle B_{j}\left\langle b^{-}\right\rangle \top\right) \rightarrow[a] B_{j} \varphi \quad(* *)
$$

Note that in $(*), B_{j}[b] \varphi$ instead of $[b] \varphi$ is not needed because we use the global notion of logical consequence $\models_{\text {EDL }}$. Now, consider $\varphi=\perp$ in $(* *)$ : $B_{j}[b] \perp$ means that perception of event $b$ was unexpected by agent $j$, while $\langle a\rangle B_{j}\left\langle b^{-}\right\rangle \top$ means that $j$ actually perceives $a$ as $b$. By our no-forgetting constraint it follows that $[a] B_{j} \perp$, i.e. unexpected events make agents go crazy. In fact, one would like to avoid agents believing inconsistencies: in such situations some sort of belief revision should take place. We do not investigate this further here. 


\subsection{Completeness}

\section{Definition 2.5 (Proof system of EDL)}

The logic EDL is defined by the multi-modal logic $\mathrm{K}$ for all the modal operators $B_{j},[a]$ and $\left[a^{-}\right]$, plus the axioms schemes Conv $_{1}$, Conv $_{2}, \mathrm{NF}, \mathrm{NL}$ and ED below:

$$
\begin{array}{ll}
\text { Conv }_{1} & \vdash_{\mathrm{EDL}} \varphi \rightarrow[a]\left\langle a^{-}\right\rangle \varphi \\
\text { Conv }_{2} & \vdash_{\mathrm{EDL}} \varphi \rightarrow\left[a^{-}\right]\langle a\rangle \varphi \\
\mathrm{NF} & \vdash_{\mathrm{EDL}} B_{j} \bigwedge_{a \in E}[a] \varphi \rightarrow \bigwedge_{a \in E}[a] B_{j} \varphi \\
\mathrm{NL} & \vdash_{\mathrm{EDL}}\langle a\rangle \hat{B}_{j}\left\langle b^{-}\right\rangle \top \rightarrow\left([a] B_{j} \varphi \rightarrow B_{j}[b] \varphi\right) \\
\mathrm{ED} & \vdash_{\mathrm{EDL}}\langle a\rangle B_{j} \varphi \rightarrow[a] B_{j} \varphi
\end{array}
$$

Conv $_{1}$ and Conv ${ }_{2}$ are the standard conversion axioms of tense logic and converse PDL. NF, NL and ED respectively axiomatize no-forgetting, no-learning and epistemic determinism.

We write $\Gamma \vdash_{\text {EDL }} \varphi$ when $\varphi$ is provable from the set of formulas $\Gamma$ in this axiomatics.

One can then show that EDL is strongly complete:

Proposition 2.6 For every set of $\mathcal{L}_{E D L}$-formulas $\Gamma$ and $\mathcal{L}_{E D L}$-formula $\varphi$,

$$
\Gamma \models_{E D L} \varphi \text { iff } \Gamma \vdash_{E D L} \varphi .
$$

PROOF. The proof follows from Sahlqvist's theorem [18]: all our axioms NF, $\mathrm{NL}$, ED are of the required form, and match the respective constraints $\mathrm{nf}, \mathrm{nl}$, ed. QED

\section{BMS: Static models, Event models, and their prod- ucts}

We here present a star-free version of Baltag's dynamic epistemic logic BMS without the iteration operator $*$ and without common belief [4, B]. We have the same sets of propositional symbols $\Phi$, agent symbols $G$ and event symbols $E$. We recall that as before, $G$ and $E$ are finite.

\subsection{Semantics}

Static models are standard epistemic models of the form $M^{s}=(W, R, V)$, where $W$ is a set of possible worlds, $R: G \rightarrow 2^{W \times W}$ assigns an accessibility relation to each agent, and $V: \Phi \rightarrow 2^{W}$ is a valuation. 
Event models are of the form $A=(E, R$, Pre $)$, where $E$ is a finite set of possible events, $R: G \rightarrow 2^{E \times E}$ assigns an accessibility relation to each agent, Pre $: E \rightarrow \mathcal{L}$ is a precondition function associating epistemic formulas to possible events.

Intuitive interpretation. Informally, $\operatorname{Pre}(a)$ is the precondition that a world must fulfill so that the event $a$ can take place in this world. For example $\operatorname{Pre}(a)=\top$ means that event $a$ can take place in any world. When we have $R_{j}(a)=\{b\}$ then the occurrence of $a$ is perceived by agent $j$ as the occurrence of $b$; when $R_{j}(a)=\left\{b_{1}, b_{2}\right\}$ then the occurrence of $a$ is perceived by agent $j$ indistinguishably as the occurrence of $b_{1}$ or $b_{2}$; etc.

Product construction. Given a static model $M^{s}=(W, R, V)$ and an event model $A=(E, R$, Pre $)$, their product $M^{s} \otimes A$ is a static model describing the situation after the event described by $A$ occurred in $M^{s}$ :

$$
M^{s} \otimes A=\left(W^{\prime}, R^{\prime}, V^{\prime}\right)
$$

where the new set of possible worlds is $W^{\prime}=\left\{(w, a) \mid M^{s}, w \models \operatorname{Pre}(a)\right\}$, the new valuation is $V^{\prime}(p)=\{(w, a) \mid w \in V(p)\}$, and the new static accessibility relation is defined by

$$
\left(w_{1}, a_{1}\right) R_{j}^{\prime}\left(w_{2}, a_{2}\right) \text { iff } w_{1} R_{j} w_{2} \text { and } a_{1} R_{j} a_{2} .
$$

While the truth condition for the epistemic operator is just as in Hintikka's epistemic logic and in EDL, the product construction gives a semantics to the $[a]$ operator which is quite different from that of PDL and EDL. It highlights that BMS is a dynamic extension of epistemic logic, while EDL is an epistemic extension of PDL.

$$
M^{s}, w \models[a] \varphi \text { iff } M^{s}, w \models \operatorname{Pre}(a) \text { implies } M^{s} \otimes A,(w, a) \models \varphi
$$

Finally, validity of $\varphi$ in BMS (noted $\models_{\mathrm{BMS}} \varphi$ ) is defined as usual as truth in every world of every BMS-model. Note that validity means validity w.r.t. a fixed event model $A$.

\subsection{Completeness}

Suppose we are given an event model $A$. The axiomatics of BMS is made up of the principles of the multi-modal logic $\mathrm{K}$ for the modal operators $B_{j}$ and $[a]$, together with the following axioms [4, 3].

$$
\begin{aligned}
& \vdash_{\text {BMS }}[a] p \leftrightarrow(\operatorname{Pre}(a) \rightarrow p) \\
& \vdash_{\mathrm{BMS}}[a] \neg \varphi \leftrightarrow(\operatorname{Pre}(a) \rightarrow \neg[a] \varphi) \\
& \vdash_{\mathrm{BMS}}[a] B_{j} \varphi \leftrightarrow\left(\operatorname{Pre}(a) \rightarrow B_{j}\left[b_{1}\right] \varphi \wedge \ldots \wedge B_{j}\left[b_{n}\right] \varphi\right) \\
& \text { where } b_{1}, \ldots, b_{n} \text { is the list of all } b \text { such that } a R_{j} b \text {. }
\end{aligned}
$$


We write $\vdash_{\text {BMS }} \varphi$ when $\varphi$ is provable from these principles. Note that this axiomatization depends on a particular event model $A$. (We might have written $\vdash_{\mathrm{BMS}}^{A} \varphi$.)

For example for every event model $A$ where $\operatorname{Pre}(a)=\top, \operatorname{Pre}(b)=p$, and $R_{j}(a)=\{b\}$ we obtain $\vdash_{\mathrm{BMS}}[a] B_{j} p$. Indeed, $\vdash_{\mathrm{BMS}}[a] B_{j} p \leftrightarrow\left(\operatorname{Pre}(a) \rightarrow B_{j}[b] p\right)$ and $\vdash_{\text {BMS }} B_{j}[b] p$ because $\vdash_{\text {BMS }}[b] p$.

\section{From BMS to EDL}

In this section we provide two embeddings of BMS into EDL: a 'semantic' one (section 4.TI) and a 'syntactic' one (section 4.2). This duality will allow us to state a representation theorem in section 4.3 relating these two equivalent characterizations of BMS in EDL.

For the syntactic embedding we will use a particular EDL-theory that encodes syntactically the structure of a given BMS event model $A$.

\section{Definition 4.1 (Theory of an event model)}

Let $A=(E, R$, Pre $)$ be an event model. The theory of $A$, written $\Gamma(A)$, is made up of the following non-logical axioms:

(1) $p \rightarrow[a] p$ and $\neg p \rightarrow[a] \neg p$, for every $a \in E$ and $p \in \Phi$;

(2) $\langle a\rangle \top \leftrightarrow \operatorname{Pre}(a)$, for every $a \in E$;

(3) $[a] B_{j}\left(\left(\left\langle a_{1}^{-}\right\rangle \top \vee \ldots \vee\left\langle a_{n}^{-}\right\rangle \top\right) \wedge\left(\left[b_{1}^{-}\right] \perp \wedge \ldots \wedge\left[b_{n}^{-}\right] \perp\right)\right)$, where $a_{1}, \ldots, a_{n}$ is the list of all $a_{i}$ such that $a_{i} \in R_{j}(a)$, and $b_{1}, \ldots, b_{n}$ is the list of all $b_{i}$ such that $b_{i} \notin R_{j}(a)$;

(4) $\hat{B}_{j} \operatorname{Pre}(b) \rightarrow[a] \hat{B}_{j}\left\langle b^{-}\right\rangle \top$, for every $(a, b)$ such that $b \in R_{j}(a)$.

Axioms 1 encode the fact that events do not change propositional facts of the world where they occur (see the definition of $V^{\prime}(p)$ in Section B.T). Axioms 2 encode the fact that an event $a$ can occur in a world iff this world satisfies the precondition of event $a$ (see the definition of $W^{\prime}$ in Section B.]). Axioms 3 encode the Kripke structure of the event model. Axioms 4 encode the definition of $R_{j}^{\prime}$ (see the definition of $R_{j}^{\prime}$ in Section B.T).

Example 4.2 Consider that $G=\{i, j\}$ and $\Phi=\{p\}$. In Figure $\square$ we recall the event models $A_{1}$ and $A_{2}$ corresponding respectively to the public announcement of $\varphi$ and the private announcement of $\varphi$ to $A$, where $\varphi \in \mathcal{L}$. Here, $\operatorname{Pre}(a)=\varphi$ in both models and $\operatorname{Pre}(b)=\top$. 


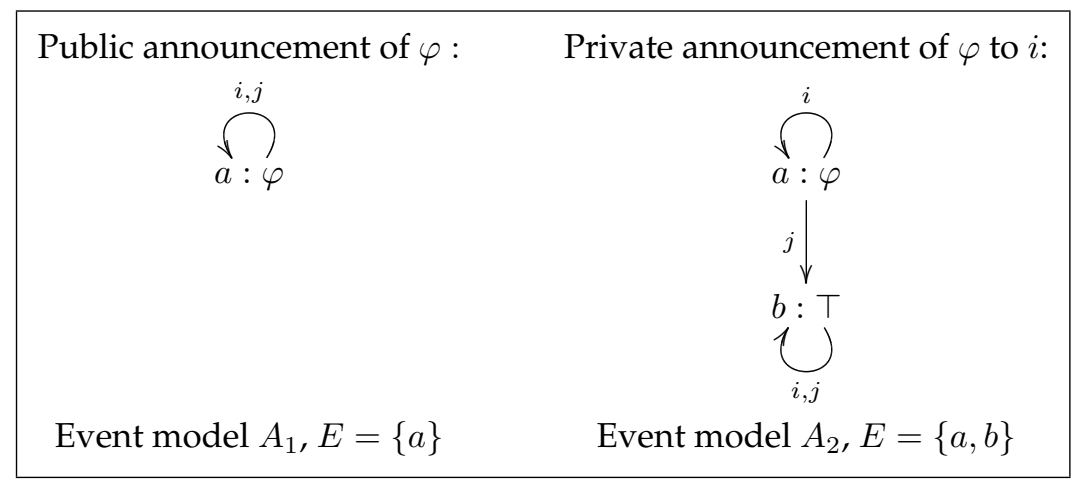

Figure 2: Event models for public announcement and private announcement

Applying Definition 4.1 , we obtain that $\Gamma\left(A_{1}\right)$ contains $p \rightarrow[a] p$ and $\neg p \rightarrow$ $[a] \neg p$ by item (1), $\langle a\rangle \top \leftrightarrow \varphi$ by item (2), $[a] B_{i}\left(\left\langle a^{-}\right\rangle \top\right),[a] B_{j}\left(\left\langle a^{-}\right\rangle \top\right), \hat{B}_{i} \varphi \rightarrow$ $[a] \hat{B}_{i}\left\langle a^{-}\right\rangle \top$ by item (4), $\hat{B}_{j} \varphi \rightarrow[a] \hat{B}_{j}\left\langle a^{-}\right\rangle \top$ by item (4).

Besides, $\Gamma\left(A_{2}\right)$ contains $p \rightarrow[a] p$ and $\neg p \rightarrow[a] \neg p, p \rightarrow[b] p$ and $\neg p \rightarrow[b] \neg p$ by item (1), $\langle a\rangle \top \leftrightarrow \varphi,\langle b\rangle \top^{\top} \leftrightarrow \top$ by item (2), $[a] B_{i}\left(\left\langle a^{-}\right\rangle \top \wedge\left[b^{-}\right] \perp\right)$, $[a] B_{j}\left(\left\langle b^{-}\right\rangle \top \wedge\left[a^{-}\right] \perp\right),[b] B_{i}\left(\left\langle b^{-}\right\rangle \top \wedge\left[a^{-}\right] \perp\right),[b] B_{j}\left(\left\langle b^{-}\right\rangle \top \wedge\left[a^{-}\right] \perp\right)$ by item (3), $\hat{B}_{i} \varphi \rightarrow[a] \hat{B}_{i}\left\langle a^{-}\right\rangle \top, \hat{B}_{i} \top \rightarrow[b] \hat{B}_{i}\left\langle b^{-}\right\rangle \top, \hat{B}_{j} \top \rightarrow[a] \hat{B}_{j}\left\langle b^{-}\right\rangle \top, \hat{B}_{j} \top \rightarrow$ $[b] \hat{B}_{j}\left\langle b^{-}\right\rangle \top$ by item (4).

\subsection{A 'semantic' embedding}

We first introduce the notion of forest generated in the BMS style by a static model and an event model (which is just as in Yap's construction [28]).

Definition 4.3 Let $M^{s}$ be a static model and $A$ an event model. We define the tuple Forest EDL $\left(M^{s}, A\right)=(W, R, \mathcal{R}, V)$ by $W=\bigcup_{n} W^{n}, V(p)=\bigcup_{n} V^{n}(p)$, $\mathcal{R}_{a}=\bigcup_{n} \mathcal{R}_{a}^{n}$, and $R_{j}=\bigcup_{n} R_{j}^{n}$, where the tuples $M^{n} \stackrel{n}{=}\left(W^{n}, R_{j}^{n}, \mathcal{R}_{a}^{n}, V^{n}\right)$ are defined inductively as follows. ${ }^{\square}$

- $M^{0}=M^{s}$

- $M^{n+1}=M^{n} \otimes_{\mathrm{EDL}} A=\left(W^{n+1}, R^{n+1}, \mathcal{R}^{n+1}, V^{n+1}\right)$ where

- $W^{n+1}=W^{n} \cup\left\{(w, a) \mid w \in W^{n}\right.$ and $\left.M^{s}, w \models \operatorname{Pre}(a)\right\} ;$

- $R_{j}^{n+1}=R_{j}^{n} \cup\left\{\left(\left(w_{1}, a_{1}\right),\left(w_{2}, a_{2}\right)\right) \mid w_{1} R_{j}^{n} w_{2}\right.$ and $\left.a_{1} R_{j} a_{2}\right\}$;

- $\mathcal{R}_{a}^{n+1}=\mathcal{R}_{a}^{n} \cup\left\{(w,(w, a)) \mid w \in W^{n}\right\} ;$

\footnotetext{
${ }^{2}$ Note that we use $\otimes_{\mathrm{EDL}}$ to distinguish our product construction here from the BMS product that we write $\otimes_{\mathrm{BMS}}$ from now on to avoid confusion.
} 


$$
\text { - } V^{n+1}(p)=V^{n}(p) \cup\left\{(w, a) \mid w \in W^{n} \text { and } w \in V^{n}(p)\right\} .
$$

Forest $_{\mathrm{EDL}}(A)$ is defined as the class of all tuples Forest EDL $\left(M^{s}, A\right)$ where $M^{s}$ is a static model.

Forest $_{\mathrm{EDL}}\left(M^{s}, A\right)$ is obviously an EDL-model and it is generated by the event model $A$. So it seems natural that the syntactic encoding $\Gamma(A)$ of this event model be true in Forest $_{\mathrm{EDL}}\left(M^{s}, A\right)$.

Proposition 4.4 Let $M^{s}$ be a static model and let $A$ be an event model. Then Forest EDL $\left(M^{s}, A\right)$ is an EDL-model and Forest $E D L\left(M^{s}, A\right) \models \Gamma(A)$.

PROOF. The proof that Forest $\mathrm{EDL}_{\mathrm{EL}}\left(M^{s}, A\right)$ is an EDL-model is standard. So we only prove the second part of the proposition. Conditions (1) and (2) of Definition 4.0 are clearly fulfilled. As for condition (3), let $w \in W^{\infty}$, then $w^{\prime}$ is such that $w \mathcal{R}_{a} w^{\prime}$ iff $w^{\prime}=(w, a)$. Now $(w, a) R_{j} u$ iff $u=(v, b)$ with $w R_{j} v$ and $a R_{j} b$ by definition of $\otimes_{\mathrm{EDL}}$. So for all $u$ such that $(w, a) R_{j} u$, there are $b$ and $v$ such that $a R_{j} b$ and $v \mathcal{R}_{b} u$. This proves that Forest $\mathrm{EDL}_{\mathrm{EL}}\left(M^{s}, A\right), w=\mathrm{EDL}[a] B_{j}\left(\left\langle a_{1}^{-}\right\rangle \top \vee \ldots \vee\right.$ $\left.\left\langle a_{n}^{-}\right\rangle \top\right)$ where $a_{1}, \ldots, a_{n}$ is the list of all $a_{i}$ such that $a R_{j} a_{i}$. Finally, concerning condition (4), assume Forest $\mathrm{EDL}_{\mathrm{EL}}\left(M^{s}, A\right), w \models_{\mathrm{EDL}} \hat{B}_{j} \operatorname{Pre}(b)$ and $w \mathcal{R}_{a}(w, a)$. Then there is $v$ such that $w R_{j} v$ and $v \mathcal{R}_{b}(v, b)$. So by definition of $\otimes_{\mathrm{EDL}}$, because $a R_{j} b$, we have $(w, a) R_{j}(v, b)$. Hence Forest $_{\mathrm{EDL}}\left(M^{s}, A\right),(w, a) \models \mathrm{EDL} \hat{B}_{j}\left\langle b^{-}\right\rangle \top$ and finally Forest $\mathrm{EDL}_{\mathrm{EL}}\left(M^{s}, A\right), w \models_{\mathrm{EDL}}[a] \hat{B}_{j}\left\langle b^{-}\right\rangle \top$. QED

By nature of the EDL setting, Forest $\operatorname{EDL}\left(M^{s}, A\right)$ explicitly represents the iterations of the BMS update product by $A$ ad infinitum, starting from the initial static model $M^{s}$. Therefore the following proposition is also not surprising.

Proposition 4.5 Let $M^{s}$ be a static model and let $A$ be an event model. Then for all $\varphi \in \mathcal{L}_{B M S}$

$$
M^{s}, w \models_{B M S} \varphi \text { iff } \text { Forest }_{E D L}\left(M^{s}, A\right), w \models_{E D L} \varphi .
$$

(We added subscripts to $=$ in order to help the reader to distinguish the two kinds of models.)

PROOF. We first prove a lemma.

Lemma 4.6 Let $k \geq 0$. Then $\left(M^{s} \otimes_{B M S} A\right)^{k},(w, a)$ is bisimilar to $M^{k+1},(w, a)$ (in notation: $\left.\left(M^{s} \otimes_{B M S} A\right)^{k},(w, a) \leftrightarrow M^{k+1},(w, a)\right)$, where $\left(M^{s} \otimes_{B M S} A\right)^{k}$ is the result of the iteration process applied $k$ times to the static model $M^{s} \otimes_{B M S} A$ and the event model $A$.

PROOF. We prove it by induction on $k$.

$k=0:\left(M^{s} \otimes_{\mathrm{BMS}} A\right)^{0}=M^{s} \otimes_{\mathrm{BMS}} A$, and $M^{1}=M^{s} \otimes_{\mathrm{EDL}} A$. Then by definition of $\otimes_{\mathrm{EDL}}$, we clearly have $\left(M^{s} \otimes_{\mathrm{BMS}} A\right)^{0},(w, a) \Leftrightarrow M^{1},(w, a)$. 


$$
\begin{aligned}
k+1: & \left(M^{s} \otimes_{\mathrm{BMS}} A\right)^{k+1}=\left(M^{s} \otimes_{\mathrm{BMS}} A\right)^{k} \otimes_{\mathrm{EDL}} A . \text { Now }\left(M^{s} \otimes_{\mathrm{BMS}} A\right)^{k},(w, a) \leftrightarrows \\
& M^{k+1},(w, a) \text { by induction hypothesis. So }\left(M^{s} \otimes_{\mathrm{BMS}} A\right)^{k} \otimes_{\mathrm{EDL}} A,(w, a) \leftrightarrows \\
& M^{k+1} \otimes_{\mathrm{EDL}} A,(w, a) \text { because for any static models } M \text { and } M^{\prime}, \text { if } \\
& M, w \leftrightarrows M^{\prime}, w^{\prime} \text { then } M \otimes_{\mathrm{EDL}} A, w \leftrightarrows M^{\prime} \otimes_{\mathrm{EDL}} A, w^{\prime} . \\
& \text { Then }\left(M^{s} \otimes_{\mathrm{BMS}} A\right)^{k+1},(w, a) \leftrightarrows M^{k+2},(w, a) .
\end{aligned}
$$

For any formula $\varphi$ we define the integer $\delta(\varphi)$ as the maximum number of nested event operator occurrences as follows:

- $\delta(p)=0$

- $\left.\delta\left(\varphi_{1} \wedge \varphi_{2}\right)=\max \left(\delta\left(\varphi_{1}\right), \delta\left(\varphi_{2}\right)\right)\right)$

- $\delta(\neg \varphi)=\delta\left(B_{j} \varphi\right)=\delta(\varphi)$

- $\delta([a] \varphi)=\delta\left(\left[a^{-}\right] \varphi\right)=\delta(\varphi)+1$

We set $\mathcal{P}(k)$ : "For all $\varphi \in \mathcal{L}_{\mathrm{BMS}}$ such that $\delta(\varphi)=k, M^{s}, w \models_{\mathrm{BMS}} \varphi$ iff $M^{k}, w=_{\operatorname{EDL}} \varphi^{\prime \prime}$, where $M^{s}$ is the static model and $M^{k}$ is the iteration of the product construction.

We prove $\mathcal{P}(k)$ for all $k$ by induction on $k$.

$k=0$ : Then $\varphi$ is epistemic so $\mathcal{P}(0)$ holds by definition of $\otimes_{\mathrm{EDL}}$.

$k+1$ : We prove it by induction on $\varphi$.

- $\varphi=[a] \varphi^{\prime}$. We have the following cases:

$M^{s}, w \models \mathrm{BMS}[a] \varphi^{\prime}$

iff if $M^{s}, w=_{\mathrm{BMS}} \operatorname{Pre}(a)$ then $M^{s} \otimes_{\mathrm{BMS}} A,(w, a) \models_{\mathrm{BMS}} \varphi^{\prime}$

iff if $M^{s}, w=_{\mathrm{BMS}} \operatorname{Pre}(a)$ then $\left(M^{s} \otimes_{\mathrm{BMS}} A\right)^{k},(w, a) \models \varphi^{\prime}$ by Induc-

tion Hypothesis because $\delta\left(\varphi^{\prime}\right) \leq k$,

iff if $M^{s}, w \models_{\mathrm{BMS}} \operatorname{Pre}(a)$ then $M^{k+1},(w, a) \models \mathrm{EDL} \varphi^{\prime}$ by Lemma 4.6

iff if $M^{k+1}, w=_{\mathrm{EDL}} \operatorname{Pre}(a)$ then $M^{k+1},(w, a) \models_{\mathrm{EDL}} \varphi^{\prime}$

iff $M^{k+1}, w=_{\mathrm{EDL}}[a] \varphi^{\prime}$ by definition of $\otimes_{\mathrm{EDL}}$

iff $M^{k+1}, w \models$ EDL $\varphi$.

- $\varphi=\varphi_{1} \wedge \varphi_{2}$ works by Induction Hypothesis.

- $\varphi=B_{j} \varphi^{\prime}$ works as well.

- $\varphi=p$ is impossible because $k+1 \geq 1$.

Then we can easily prove that for all $\varphi$ such that $\delta(\varphi)=k, M^{k}, w \models_{E D L} \varphi$ iff Forest $\operatorname{EDL}_{\mathrm{EL}}\left(M^{s}, A\right), w \models_{\mathrm{EDL}} \varphi$. Then for all $k$, for all $\varphi$ such that $\delta(\varphi)=k$, $M^{s}, w \models_{\mathrm{BMS}} \varphi$ iff Forest $_{\mathrm{EDL}}\left(M^{s}, A\right), w \models_{\mathrm{EDL}} \varphi$, i.e. for all $\varphi \in \mathcal{L}_{\mathrm{BMS}}, M^{s}, w \models_{\mathrm{BMS}}$ $\varphi$ iff Forest $_{\mathrm{EDL}}\left(M^{s}, A\right), w=\mathrm{EDL} \varphi$.

QED

As a corollary of Proposition 4.5 we get the following 'semantic' embedding of BMS into EDL. 
Theorem 4.7 Let $A$ be an event model, and let $\varphi \in \mathcal{L}_{B M S}$. Then

$$
\models_{B M S} \varphi \text { iff } \text { Forest }_{E D L}(A) \models_{E D L} \varphi \text {. }
$$

This theorem illustrates formally the intuition that the fragment of the class of EDL-models that embeds the BMS semantics is the class of EDL-models Forest $_{\mathrm{EDL}}(A)$.

\subsection{A 'syntactic' embedding}

In this section we prove that $\Gamma(A)$ correctly encodes the event model $A$ from a syntactic point of view, in the sense that for every formula $\varphi \in \mathcal{L}_{\mathrm{BMS}}$,

$$
\vdash_{\mathrm{BMS}} \varphi \operatorname{iff} \Gamma(A) \vdash_{\mathrm{EDL}} \varphi . \quad(* * *)
$$

To do so, we first prove that the axiom of determinism stated in the following proposition is a logical consequence of $\Gamma(A)$ in EDL. This is comforting because the axiom of determinism is indeed valid in BMS .

Proposition 4.8 Let $A$ be an event model. For every $\varphi \in \mathcal{L}_{B M S}$ we have $\Gamma(A) \models_{E D L}$ $\langle a\rangle \varphi \rightarrow[a] \varphi$.

Proof. Let $A=(E, R$, Pre $)$ be a given event model, and let $M$ be an EDLmodel such that $M \models \psi$ for every $\psi \in \Gamma(A)$. Assume $w_{0} \mathcal{R}_{a} v_{0}$ and $w_{0} \mathcal{R}_{a} u_{0}$ with $v_{0} \neq u_{0}$. We are going to show that $u_{0}$ and $v_{0}$ are bisimilar.

$Z^{e}$ is defined to be an epistemic bisimulation between models $M_{1}$ and $M_{2}$ if $Z^{e}$ is a bisimulation between the restriction of these models to epistemic accessibility relations. Let $Z^{e}:=\{(w, w): w \in W\} \cup\left\{\left(v_{0}, u_{0}\right)\right\}$. We are going to show that $Z^{e}$ is an epistemic bisimulation. To do so, we need to prove

1. $u_{0} \in V(p)$ iff $v_{0} \in V(p)$ for all $p \in \Phi$;

2. if $v_{0} R_{j} v^{\prime}$ then $u_{0} R_{j} v^{\prime}$;

3. if $u_{0} R_{j} u^{\prime}$ then $v_{0} R_{j} u^{\prime}$.

(1) is guaranteed by the first item of Definition 4.7. (2) and (3) are guaranteed by epistemic determinism: ed makes that $R_{j}\left(u_{0}\right)=R_{j}\left(v_{0}\right)$.

Now from $Z^{e}$, we are going to build up a bisimulation. We proceed as follows.

$Z^{0}=Z^{e}$;

$Z^{n+1}=\left\{\left(u_{n+1}, v_{n+1}\right) \mid u_{n} \mathcal{R}_{a} u_{n+1}\right.$ and $v_{n} \mathcal{R}_{a} v_{n+1}$ for some $a \in E$ and $\left.u_{n} Z^{n} v_{n}\right\} ;$

$Z=\bigcup_{n \in \mathbb{N}} Z^{n}$.

We are going to show that $Z$ is a bisimulation.

1. We first show that $Z$ is an epistemic bisimulation: we prove by induction on $n$ that every $Z^{n}$ is an epistemic bisimulation.

We have already proved that $Z^{0}$ is an epistemic bisimulation. Assume it is true for $Z^{n}$ and $u_{n+1} Z^{n+1} v_{n+1}$. Then there are $u_{n}, v_{n}$ such that $u_{n} Z^{n} v_{n}$, $u_{n} \mathcal{R}_{a} u_{n+1}$ and $v_{n} \mathcal{R}_{a} v_{n+1}$. 
(a) $u_{n} \in V(p)$ iff $v_{n} \in V(p)$ because $Z^{n}$ is an epistemic bisimulation. So $u_{n+1} \in V(p)$ iff $v_{n+1} \in V(p)$ by Definition 4 . 1 (1).

(b) Assume $u_{n+1}^{\prime} \in R_{j}\left(u_{n+1}\right)$. Then by nf, there are $u_{n}^{\prime}$ and $b$ such that $u_{n}^{\prime} \in R_{j}\left(u_{n}\right)$ and $u_{n+1}^{\prime} \in \mathcal{R}_{b}\left(u_{n}^{\prime}\right)$.

Then there is $v_{n}^{\prime} \in W$ such that $v_{n}^{\prime} \in R_{j}\left(v_{n}\right)$ and $v_{n}^{\prime} Z^{n} u_{n}^{\prime}$ by induction hypothesis. But $M, u_{n}^{\prime} \models \operatorname{Pre}(b)$ because $M, u_{n}^{\prime} \models\langle b\rangle \top$ and Definition 4.] (2). Besides for all $\varphi \in \mathcal{L}, M, v_{n}^{\prime}=\varphi$ iff $M, u_{n}^{\prime} \mid=\varphi$ because $Z^{n}$ is an epistemic bisimulation by induction hypothesis. So $M, v_{n}^{\prime} \models \operatorname{Pre}(b)$ because $\operatorname{Pre}(b) \in \mathcal{L}$.

Then there is $v_{n+1}^{\prime}$ such that $v_{n+1}^{\prime} \in \mathcal{R}_{b}\left(v_{n}^{\prime}\right)$ by Definition 4.1] (2). So $v_{n+1}^{\prime} \in\left(R_{j} \circ \mathcal{R}_{b}\right)\left(v_{n}\right)$.

Besides $M, u_{n}=\hat{B}_{j} \operatorname{Pre}(b)$, so $M, v_{n}=\hat{B}_{j} \operatorname{Pre}(b)$ by induction hypothesis and because $\hat{B}_{j} \operatorname{Pre}(b) \in \mathcal{L}$. So $M, v_{n}=[a] \hat{B}_{j}\left\langle b^{-}\right\rangle \top$ by Definition 4.7 (4).

But $M, v_{n}=\langle a\rangle \top$, so $M, v_{n}=\langle a\rangle \hat{B}_{j}\left\langle b^{-}\right\rangle \top$. So $\left(\mathcal{R}_{a} \circ R_{j} \circ \mathcal{R}_{b}^{-1}\right)\left(v_{n}\right) \neq$ $\emptyset$. So $\left(R_{j} \circ \mathcal{R}_{b}\right)\left(v_{n}\right) \subseteq\left(\mathcal{R}_{a} \circ R_{j}\right)\left(v_{n}\right)$ by nl. So there is $v_{n+1}^{\prime \prime} \in \mathcal{R}_{a}\left(v_{n}\right)$ such that $v_{n+1}^{\prime} \in R_{j}\left(v_{n+1}^{\prime \prime}\right)$. Then by ed, $v_{n+1}^{\prime} \in R_{j}\left(v_{n+1}\right)$.

Besides $u_{n}^{\prime} Z^{n} v_{n}^{\prime}$ and $u_{n+1}^{\prime} \in \mathcal{R}_{b}\left(u_{n}^{\prime}\right), v_{n+1}^{\prime} \in \mathcal{R}_{b}\left(v_{n}^{\prime}\right)$.

So by definition of $Z^{n+1}, u_{n+1}^{\prime} Z^{n+1} v_{n+1}^{\prime}$.

So there is $v_{n+1}^{\prime}$ such that $v_{n+1}^{\prime} \in R_{j}\left(v_{n+1}\right)$ and $u_{n+1}^{\prime} Z^{n+1} v_{n+1}^{\prime}$

(c) The case $v_{n+1}^{\prime} \in R_{j}\left(v_{n+1}\right)$ is similar.

So for all $n \in \mathbb{N}, Z^{n}$ is an epistemic bisimulation. Henceforth $Z$ is also an epistemic bisimulation.

2. Now we are going to show that $Z$ is a full bisimulation. Assume $u Z v$ for some $u, v \in W$. Then $u Z^{n} v$ for some $n \in \mathbb{N}$.

(a) If $u^{\prime} \in \mathcal{R}_{a}(u)$ then $M, u \models \operatorname{Pre}(a)$ by Definition 4.1 (2). So $M, v \models$ $\operatorname{Pre}(a)$ because $Z$ is an epistemic bisimulation and $\operatorname{Pre}(a) \in \mathcal{L}^{C}$.

So there is $v^{\prime}$ such that $v \mathcal{R}_{a} v^{\prime}$. But then $u^{\prime} Z^{n+1} v^{\prime}$ by construction of $Z^{n}$. So $u^{\prime} Z v^{\prime}$.

(b) Similarly we prove that if $v^{\prime} \in \mathcal{R}_{a}(v)$ then there is $u^{\prime}$ such that $u^{\prime} \in$ $\mathcal{R}_{a}(u)$ and $u^{\prime} Z v^{\prime}$.

QED

Now, we prove the two directions of $(* * *)$ by means of two propositions.

Proposition 4.9 Let $A$ be an event model, and let $\psi \in \mathcal{L}_{B M S}$. If $\nvdash_{B M S} \psi$ then $\Gamma(A) \not \forall_{E D L} \psi$. 
ProOF. We have to prove that if there is a static model $M^{s}$ and a $w$ in $M^{s}$ such that $M^{s}, w \forall \psi \psi$ then $M^{s}$ can be turned into an EDL-model $M$ such that $M \models \Gamma(A)$ and $M, w^{\prime} \not \models \psi$ for some $w^{\prime}$ of $M$. We naturally consider the EDLmodel $M=$ Forest $_{\mathrm{EDL}}\left(M^{s}, A\right)$ and $w^{\prime}=w$.

By Proposition 4.4 we have Forest $\mathrm{EDL}\left(M^{s}, A\right) \models \Gamma(A)$.

By Proposition 4.5 we have Forest $\mathrm{EDL}\left(M^{s}, A\right), w \not \models \psi$.

QED

Proposition 4.10 Let $A$ be an event model, and let $\psi \in \mathcal{L}_{B M S}$. If $\models_{B M S} \psi$ then $\Gamma(A) \models E D L \psi$.

PROOF. We take advantage of the complete axiomatization of BMS-validities given in [4, 3], and show that the BMS-axioms are EDL-valid, and that the BMS-inference rules preserve EDL-validity. As the inference rules of BMS and EDL are identical (i.e. modus ponens and necessitation) it is clear that the BMSinference rules preserve EDL-theoremhood. It is straightforward to show that every instance of the BMS-axioms not involving dynamic operators is EDLvalid. So what remains is to prove that the BMS schemas

$$
\begin{array}{ll}
\text { R1 } & {[a] p \leftrightarrow(\operatorname{Pre}(a) \rightarrow p)} \\
\text { R2 } & {[a] \neg \varphi \leftrightarrow(\operatorname{Pre}(a) \rightarrow \neg[a] \varphi)} \\
\text { R3 } & {[a] B_{j} \varphi \leftrightarrow\left(\operatorname{Pre}(a) \rightarrow B_{j}\left[a_{1}\right] \varphi \wedge \ldots \wedge B_{j}\left[a_{n}\right] \varphi\right)}
\end{array}
$$

where $a_{1}, \ldots, a_{n}$ is the list of all $a_{i}$ such that $a R_{j} a_{i}$, are logical consequences of $\Gamma(A)$ in EDL.

R1 Axiom R1 can be proved by the nonlogical axioms (1) $p \rightarrow[a] p$ and (2) $\langle a\rangle \top \leftrightarrow \operatorname{Pre}(a)$ of the theory $\Gamma(A)$ in Definition 4.1 .

R2 For the left-to-right direction of R2 we have

$$
\Gamma(A) \models_{\mathrm{EDL}}([a] \neg \varphi \wedge \operatorname{Pre}(a) \wedge[a] \varphi) \rightarrow \perp
$$

because of the nonlogical axiom (2) $\langle a\rangle \top \leftrightarrow \operatorname{Pre}(a)$ of Definition 4.7 .

For the right-to-left direction, on the one hand we have $\Gamma(A) \models_{\mathrm{EDL}} \neg \operatorname{Pre}(a) \rightarrow$ $[a] \perp$ again by the nonlogical axiom (2) of Definition 4.1, and on the other hand $\Gamma(A) \models$ EDL $\neg[a] \varphi \rightarrow[a] \neg \varphi$ by Proposition 4.8 .

R3 For the left-to-right direction of R3, let $M$ be an EDL-model such that $M=_{\mathrm{EDL}} \Gamma(A)$ and suppose

$$
M, w \models \mathrm{EDL}[a] B_{j} \varphi \wedge \operatorname{Pre}(a),
$$

and suppose $M, w \models_{\mathrm{EDL}} \neg B_{j}[b] \varphi$ for some $b$ such that $a R_{j} b$. So there must exist worlds $w^{\prime}$ and $v^{\prime}$ such that $w R_{j} w^{\prime}, w^{\prime} \mathcal{R}_{b} v^{\prime}$ and $M, v^{\prime} \models \neg \varphi$. Therefore $M, w^{\prime} \models \operatorname{Pre}(b)$ by nonlogical axiom 4.$]$ (2), and $M, w \models_{\mathrm{EDL}}$ $\hat{B}_{j} \operatorname{Pre}(b)$. As $a R_{j} b$, our nonlogical axiom $4 . \mathbb{D}$ (4) tells us that $M, w \models_{\mathrm{EDL}}$ $\hat{B}_{j} \operatorname{Pre}(b) \rightarrow[a] \hat{B}_{j}\left\langle b^{-}\right\rangle \top$, and hence $M, w \models$ EDL $[a] \hat{B}_{j}\left\langle b^{-}\right\rangle \top$. As by hypothesis $M, w \mid=\mathrm{EDL} \operatorname{Pre}(a)$, by nonlogical axiom 4.$](2)\left(\mathcal{R}_{a} \circ R_{j} \circ \mathcal{R}_{b}^{-1}\right)(w) \neq$ $\emptyset$. By the constraint $\mathrm{nl}$ on EDL-models we have

$$
\left(R_{j} \circ \mathcal{R}_{b}\right)(w) \subseteq\left(\mathcal{R}_{a} \circ R_{j}\right)(w),
$$


i.e. $v^{\prime} \in\left(\mathcal{R}_{a} \circ R_{j}\right)(w)$. As we have supposed that $M, w \models_{\mathrm{EDL}}[a] B_{j} \varphi$, we must have $M, v^{\prime} \models_{\operatorname{EDL}} \varphi$, which is contradictory.

For the right-to-left direction of $\mathrm{R} 3$, we know that $\Gamma(A) \models$ EDL $\neg \operatorname{Pre}(a) \rightarrow$ $[a] \perp$ again by the nonlogical axiom $4 . \rrbracket$ (2), so it remains to prove that

$$
\Gamma(A) \models_{\mathrm{EDL}}\left(B_{j}\left[a_{1}\right] \varphi \wedge \ldots \wedge B_{j}\left[a_{n}\right] \varphi\right) \rightarrow[a] B_{j} \varphi .\left(^{*}\right)
$$

where $a_{1}, \ldots, a_{n}$ is the list of all $a_{i}$ such that $a R_{j} a_{i}$. Suppose $M, w \models$ EDL $B_{j}\left[a_{1}\right] \varphi \wedge \ldots \wedge B_{j}\left[a_{n}\right] \varphi$, and suppose $M, w \models_{\mathrm{EDL}} \neg[a] B_{j} \varphi$. The latter implies that there are worlds $v$ and $v^{\prime}$ such that $w \mathcal{R}_{a} v R_{j} v^{\prime}$ and $M, v^{\prime} \models_{\text {EDL }}$ $\neg \varphi$. By the constraint nf, there is $b \in E$ such that $v^{\prime} \in R_{j} \circ \mathcal{R}_{b}(w)$.

Now, by the nonlogical axiom 4.7 (3) we have

$$
[a] B_{j}\left(\left(\left\langle a_{1}^{-}\right\rangle \top \vee \ldots \vee\left\langle a_{n}^{-}\right\rangle \top\right) \wedge\left(\left[b_{1}^{-}\right] \perp \wedge \ldots \wedge\left[b_{n}^{-}\right] \perp\right)\right),
$$

where $a_{1}, \ldots, a_{n}$ is the list of all $a_{i}$ such that $a_{i} \in R_{j}(a)$ and $b_{1}, \ldots, b_{n}$ is the list of all $b$ such that $b_{i} \notin R_{j}(a)$. Hence $M, v^{\prime} \models_{\text {EDL }}\left(\left\langle a_{1}^{-}\right\rangle \top \vee \ldots \vee\right.$ $\left.\left\langle a_{n}^{-}\right\rangle \top\right) \wedge\left(\left[b_{1}^{-}\right] \perp \wedge \ldots \wedge\left[b_{n}^{-}\right] \perp\right)$. So $b \in R_{j}(a)$. Then $M, w \models \operatorname{EDL} B_{j}[b] \varphi$ by $\left(^{*}\right)$. So $M, v^{\prime}=\operatorname{EDL} \neg \varphi$, which is contradictory.

QED

Putting these two results together we obtain the following 'syntactic' embedding of BMS into EDL.

Theorem 4.11 Let $A$ be an event model, and let $\varphi \in \mathcal{L}_{B M S}$. Then

$$
\vdash_{B M S} \varphi \quad \text { iff } \Gamma(A) \vdash_{E D L} \varphi
$$

PROOF. Follows easily from Propositions 4.9 and 4.10 by soundness and completeness of BMS and EDL.

QED

This theorem also provides another syntactic characterization of the BMS validities. This syntactic characterization is just made of $\Gamma(A)$ together with the axiomatization of EDL.

\subsection{A representation theorem}

Theorems 4.7 and $4 . T 7$ give us two characterizations of the BMS logic within

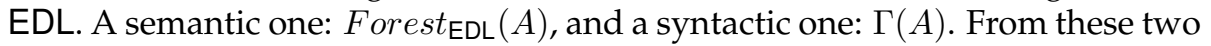
results we get easily the following representation theorem.

Theorem 4.12 Let $M$ be an EDL-model and $A$ be an event model.

$$
M=\Gamma(A) \text { iff } M \text { is bisimilar to some EDL-model of Forest }{ }_{E D L}(A) .
$$

ProOF. The right to left direction follows from Proposition 4.4. The left to right direction follows easily from Theorems 4.7 and 4.11 . 


\section{Comparison with ETL and other related work}

Another approach studying information change over time is Epistemic Temporal Logic ETL [14] (or equivalently interpreted systems [8] as shown by Pacuit [13]]). In this section we are going to compare EDL with ETL from the standpoint of [13, 24, 21] where converse events are introduced as well. We will also study their relationships with the BMS framework and some of its extensions.

\subsection{Basics of ETL}

Let $\Sigma$ be any set. Elements of $\Sigma$ are called events, and elements of the set of finite strings $\Sigma^{*}$ histories. For any two sets $X$ and $Y, X Y$ is the set of sequences consisting of an object in $X$ followed by one in $Y$. Given $h \in \Sigma^{*}$, the length of $h(\operatorname{len}(h))$ is the number of events in $h$. Given $h, h^{\prime} \in \Sigma^{*}$, we write $h \preceq h^{\prime}$ if $h$ is a prefix of $h^{\prime}$. Let $\lambda$ be the empty string. For a set of histories $\mathcal{H} \subseteq \Sigma^{*}$, FinPre $_{-\lambda}(\mathcal{H})=\left\{h \mid h\right.$ is non-empty and there is $h^{\prime} \in \mathcal{H}$ such that $\left.h \preceq h^{\prime}\right\}$. Given an event $a \in \Sigma$, we write $h \prec_{a} h^{\prime}$ if $h^{\prime}=h a$.

Definition 5.1 Let $\Sigma$ be any set of events. A protocol is a set $\mathcal{H} \subseteq \Sigma^{*}$ with FinPre $_{-\lambda}(\mathcal{H}) \subseteq \mathcal{H}$. An $E T L$ - model is a tuple $(\Sigma, \mathcal{H}, R, V)$ where $\Sigma$ is a finite set of events, $\mathcal{H}$ is a protocol, $R: G \rightarrow 2^{\mathcal{H} \times \mathcal{H}}$ assigns an accessibility relation $R(j)=R_{j}$ to each agent $j \in G$, and $V: \Phi \rightarrow 2^{\mathcal{H}}$ is a valuation.

So note that in an ETL-model events are deterministic which is not necessarily the case in an EDL-model. The language of ETL is the same as the language $\mathcal{L}_{\text {EDL }}$ of EDL. Truth conditions are defined as usual and we only recall those for the temporal operators.

- $h \models[a] \varphi$ iff $h^{\prime}=h a \in \mathcal{H}$ and $h^{\prime}=\varphi$.

- $h \models\left[a^{-}\right] \varphi$ iff $h=h^{\prime} a$ for some $h^{\prime} \in \mathcal{H}$ and $h^{\prime} \models \varphi$.

ETL-models might satisfy additional constraints listed below.

Definition 5.2 Let $T=(\Sigma, \mathcal{H}, R, V)$ be an ETL-model. $T$ satisfies:

- Propositional Stability iff for all $h \in \mathcal{H}, a \in \Sigma, h \models p$ iff $h a \models p$;

- Perfect Recall iff for all $h, h^{\prime \prime} \in \mathcal{H}, a \in \Sigma$ such that $h a \in \mathcal{H}$ and $h^{\prime \prime} \in R_{j}(h a)$ there is $h^{\prime} \in R_{j}(h)$ and $a^{\prime} \in \Sigma$ such that $h^{\prime \prime}=h^{\prime} a^{\prime}$; , B]

- No Miracles iff for all $h, h^{\prime} \in \mathcal{H}, a, a^{\prime} \in \Sigma$ with $h a, h^{\prime} a^{\prime} \in \mathcal{H}$, if there are $h^{\prime \prime}, h^{\prime \prime \prime} \in \mathcal{H}$ with $h^{\prime \prime} a, h^{\prime \prime \prime} a^{\prime} \in \mathcal{H}$ such that $h^{\prime \prime \prime} a^{\prime} \in R_{j}\left(h^{\prime \prime} a\right)$ and $h^{\prime} \in R_{j}(h)$, then $h^{\prime} a^{\prime} \in R_{j}(h a)$;

\footnotetext{
${ }^{3}$ Note that this definition of perfect recall taken from [23] is slightly different from the definition of perfect recall in $[24,121]$.
} 
- Weak No Miracles iff for all $h, h^{\prime} \in \mathcal{H}, a, a^{\prime} \in \Sigma$ with $h a, h^{\prime} a^{\prime} \in \mathcal{H}$, if there is $h^{\prime \prime} \in \mathcal{H}$ with $h^{\prime \prime} a^{\prime} \in \mathcal{H}$ such that $h^{\prime \prime} a^{\prime} \in R_{j}(h a)$ and $h^{\prime} \in R_{j}(h)$, then $h^{\prime} a^{\prime} \in R_{j}(h a)^{, \mathbb{I}}$

- Bisimulation invariance iff for all epistemically bisimilar $h, h^{\prime} \in \mathcal{H}$, if $h a \in$ $\mathcal{H}$ then $h^{\prime} a \in \mathcal{H}$.

Now, given a static model $M^{s}$ and an event model $A$, one can naturally define an ETL-model generated in the BMS style, very similarly to the way we defined an EDL-model generated in the BMS style in Definition 4.3.

Definition 5.3 Let $M^{s}=(W, R, V)$ be a static model and $A=(E, R$, Pre $)$ be an event model. We define the ETL-model Forest ETL $\left(M^{s}, A\right)=(\Sigma, \mathcal{H}, R, V)$ as follows.

- $\Sigma=W \cup E$;

- $\mathcal{H} \subseteq W E^{*}$ and $w a_{1} \ldots a_{n} \in \mathcal{H}$ iff $\left.\left(\left(w, a_{1}\right), \ldots\right), a_{n}\right) \in W^{n}$;

- $w^{\prime} a_{1}^{\prime} \ldots a_{n}^{\prime} \in R_{j}\left(w a_{1} \ldots a_{n}\right)$ iff $\left.\left.\left(\left(w^{\prime}, a_{1}^{\prime}\right), \ldots\right), a_{n}^{\prime}\right) \in R_{j}^{n}\left(\left(\left(w, a_{1}\right), \ldots\right), a_{n}\right)\right)$;

- $w a_{1} \ldots a_{n} \in V(p)$ iff $\left.\left(\left(w, a_{1}\right), \ldots\right), a_{n}\right) \in V^{n}(p)$.

The following representation theorem sets some connections between ETL and BMS. It is the counterpart in ETL of our representation Theorem 4.T2.

Theorem 5.4 [23] An ETL-model $T$ is of the form Forest ${ }_{E T L}\left(M^{s}, A\right)$ for some static model $M^{s}$ and some event model $A$ iff $T$ satisfies propositional stability, perfect recall, no miracles and bisimulation invariance.

However, the right to left direction of this theorem does not hold in general if we use the standard BMS framework [4, 3] used in this paper (in particular if we assume that $T$ is infinite). Indeed to prove this theorem, the preconditions of the event model $A$ might involve infinite conjunctions and disjunctions of epistemic formulae and not a single epistemic formula as in our paper and in $[4,3]$. We are going to need this assumption in the 2 nd item of Definition 4.1 .

\subsection{ETL and EDL}

To compare EDL and ETL we need a notion of 'equivalence' between EDLmodels and ETL-models. It is captured here formally by the notion of DTbisimulation defined as follows.

\footnotetext{
${ }^{4}$ This notion of weak no miracles is only introduced in our paper.
} 
Definition 5.5 Let $M=(W, R, \mathcal{R}, V)$ be an EDL-model and $T=(\Sigma, \mathcal{H}, R, V)$ be an ETL-model. Let $Z$ be a relation between $W$ and $\mathcal{H}$. We define the property of $Z$ being a DT-bisimulation in $w \in W$ and $h \in \mathcal{H}$, noted $Z: M, w \biguplus_{\mathrm{DT}}$ $T, h$, as follows:

- If $w Z h$ then for all $p \in \Phi, w \in V(p)$ iff $h \in V(p)$.

- If $w Z h$ and $w^{\prime} \in R_{j}(w)$ then there exists $h^{\prime} \in R_{j}(h)$ such that $w^{\prime} Z h^{\prime}$.

- If $w Z h$ and $h^{\prime} \in R_{j}(h)$ then there exists $w^{\prime} \in R_{j}(w)$ such that $w^{\prime} Z h^{\prime}$.

- If $w Z h$ and $w^{\prime} \in \mathcal{R}_{a}(w)$ then there exists $h^{\prime} \in \mathcal{H}$ such that $h \prec_{a} h^{\prime}$ and $w^{\prime} Z h^{\prime}$.

- If $w Z h$ and $h^{\prime} \in \mathcal{H}$ is such that $h \prec_{a} h^{\prime}$ then there exists $w^{\prime} \in \mathcal{R}_{a}(w)$ such that $w^{\prime} Z h^{\prime}$.

We say that $M, w$ and $T, h$ are DT-bisimilar, noted $M, w \uplus_{\mathrm{DT}} T, h$ iff there is a relation $Z$ such that $Z: M, w \biguplus_{\mathrm{DT}} T, h$.

Naturally, two 'equivalent' models satisfy the same formulas:

Proposition 5.6 Let $M$ be an EDL-model and $T$ be an ETL-model, $w \in M$ and $h \in T$. If $M, w \uplus_{D T} T, h$ then for all $\varphi \in \mathcal{L}_{E D L}, M, w=\varphi$ iff $T, h \models \varphi$.

We can now express formally that Forest $_{\text {EDL }}$ and Forest ${ }_{\text {ETL }}$ are 'equivalent' constructions.

Proposition 5.7 Let $M^{s}$ be a static model and $A$ be an event model.

$$
\text { Forest }_{E D L}\left(M^{s}, A\right) \leftrightarrows_{D T} \text { Forest }_{E T L}\left(M^{s}, A\right)
$$

PROOF. Follows easily from the definition of Forest $_{\mathrm{ETL}}\left(M^{s}, A\right) . \quad$ QED

This ends our mathematical preliminaries for the comparison of EDL and ETL. Now, natural questions to ask are: given an ETL-model, can we find an 'equivalent' EDL-model? And vice versa: given an EDL-model, can we find an 'equivalent' ETL-model? The answers to both questions are negative: first because an ETL-model does not necessarily satisfy the no-forgetting and nolearning principles; second, because an EDL-model does not necessarily satisfy the determinism principle, that is to say $\mathcal{R}_{a}$ and $\mathcal{R}_{a}^{-1}$ are partial functions for all events $a$. Nevertheless we have the following proposition.

Proposition 5.8 Any ETL-model satisfying perfect recall and weak no miracles is $D T$-bisimilar to an EDL-model satisfying determinism, and vice versa.

ProOF. We just give the corresponding ETL- and EDL-models. The proof that they satisfy perfect recall, weak no miracles and determinism is routine.

Let $M=(W, R, \mathcal{R}, V)$ be an EDL-model satisfying determinism, and let $w \in W$. We define the corresponding ETL-model $T=(\Sigma, \mathcal{H}, R, V)$ as follows. 
- $\Sigma=E$;

- $\mathcal{H}=\bigcup_{n} \mathcal{H}_{n}$ where

- $\mathcal{H}_{0}=\left\{v \mid v \in\left(\bigcup_{j \in G} R_{j}\right)^{*}(w)\right\}$

- $\mathcal{H}_{n}=\left\{w a_{1} \ldots a_{n} \mid a_{1}, \ldots, a_{n} \in \Sigma\right.$ and $\left.\left(\mathcal{R}_{a_{1}} \circ \ldots \circ \mathcal{R}_{a_{n}}\right)(w) \neq \emptyset\right\}$ for $n \geq 1$;

- $w a_{1} \ldots a_{n} \in R_{j}\left(w b_{1} \ldots b_{m}\right)$ iff $n=m$ and $v_{n} \in R_{j}\left(u_{m}\right)$, where $v_{n}=$ $\left(\mathcal{R}_{a_{1}} \circ \ldots \circ \mathcal{R}_{a_{n}}\right)(w)$ and $u_{m}=\left(\mathcal{R}_{b_{1}} \circ \ldots \circ \mathcal{R}_{b_{m}}\right)(w)$;

- $V(p)=\left\{w a_{1} \ldots a_{n} \in \mathcal{H} \mid\left(\mathcal{R}_{a_{1}} \circ \ldots \circ \mathcal{R}_{a_{n}}\right)(w) \in V(p)\right\}$, for all $p \in \Phi$.

We write $v_{n}=\left(R_{a_{1}} \circ \ldots \circ R_{a_{n}}\right)(w)$ instead of $\left\{v_{n}\right\}=\left(R_{a_{1}} \circ \ldots \circ R_{a_{n}}\right)(w)$. This makes sense because $M$ satisfies determinism.

Let $T=(\Sigma, \mathcal{H}, R, V)$ be an ETL-model. We define the corresponding EDLmodel $M=\left(W, R^{\prime}, \mathcal{R}, V^{\prime}\right)$ as follows. $W=\mathcal{H} ; R^{\prime}=R ; h^{\prime} \in \mathcal{R}_{a}(h)$ iff $h \prec_{a} h^{\prime}$; and $V^{\prime}=V$.

QED

In fact, note that perfect recall is the ETL-version of our no-forgetting principle and weak no miracles is the ETL-version of our no learning principle.

Now we are going to compare the relationships that ETL and EDL entertain with BMS. On the one hand, an EDL-model $M$ validates the BMS logic if $M \models$ $\Gamma(A)$ for some event model $A$. On the other hand, an ETL-model validates the BMS logic if it satisfies propositional stability, perfect recall, no miracles and bisimulation invariance (according to Theorem 5.4). The following proposition relates these two conditions.

Proposition 5.9 Let $M$ be an EDL-model and let $A$ be an event model. If $M \models \Gamma(A)$ then $M$ is DT-bisimilar to an ETL-model satisfying propositional stability, perfect recall, no miracles and bisimulation invariance.

Proof. Let $M=(W, R, \mathcal{R}, V)$ be an EDL-model and $w \in W$. We define the ETL-model $T=\left(\Sigma, \mathcal{H}, R^{\prime}, V^{\prime}\right)$ as in the proof of Proposition 5.8. The definition makes sense because if $M \models \Gamma(A)$ then $M$ satisfies determinism by Proposition 4.8. We now have to check that $T$ satisfies propositional stability, perfect recall, no miracles and bisimulation invariance.

Propositional stability holds because of the first item of Definition 4.]. Perfect recall holds by Proposition 5.8. We now check that no miracles and bisimulation invariance hold.

No Miracles Let $h, h^{\prime} \in \mathcal{H}, a, b \in \Sigma$ with $h a, h^{\prime} b \in \mathcal{H}$. Assume there are $h^{\prime \prime}, h^{\prime \prime \prime} \in \mathcal{H}$ with $h^{\prime \prime} a, h^{\prime \prime \prime} b \in \mathcal{H}$ such that $h^{\prime \prime \prime} b \in R_{j}\left(h^{\prime \prime} a\right)$ and $h^{\prime} \in R_{j}(h)$. 
Then $h=w a_{1} \ldots a_{n}, h^{\prime}=w a_{1}^{\prime} \ldots a_{n}^{\prime}, h^{\prime \prime}=w a_{1}^{\prime \prime} \ldots a_{m}^{\prime \prime}, h^{\prime \prime \prime}=w a_{1}^{\prime \prime \prime} \ldots a_{m}^{\prime \prime \prime}$. Let $u=\mathcal{R}_{a_{1}} \circ \ldots \circ \mathcal{R}_{a_{n}}(w) \in W, u^{\prime}=\mathcal{R}_{a_{1}^{\prime}} \circ \ldots \circ \mathcal{R}_{a_{n}^{\prime}}(w) \in W, u^{\prime \prime}=$ $\mathcal{R}_{a_{1}^{\prime \prime}} \circ \ldots \circ \mathcal{R}_{a_{m}^{\prime \prime}}(w) \in W$ and $u^{\prime \prime \prime}=\mathcal{R}_{a_{1}^{\prime \prime \prime}} \circ \ldots \circ \mathcal{R}_{a_{m}^{\prime \prime \prime}}(w) \in W$.

Then we have (1) $u^{\prime} \in R_{j}(u)$, and (2) $u^{\prime \prime \prime} \in \mathcal{R}_{a} \circ R_{j} \circ \mathcal{R}_{b}^{-1}\left(u^{\prime \prime}\right)$. We have to show that $h^{\prime} b \in R_{j}(h a)$, i.e. $u^{\prime} \in \mathcal{R}_{a} \circ R_{j} \circ \mathcal{R}_{b}^{-1}(u)$. By (2) we have that $\mathcal{R}_{a} \circ R_{j} \circ \mathcal{R}_{b}^{-1}\left(u^{\prime \prime}\right) \neq \emptyset$. Therefore $M, u^{\prime \prime} \models[a] \hat{B}_{j}\left\langle b^{-}\right\rangle \top$. So $b \in R_{j}(a)$ by Definition 4.] (3). Now, $h^{\prime} b \in \mathcal{H}$, so $\mathcal{R}_{b}\left(u^{\prime}\right) \neq \emptyset$. Therefore $M, u^{\prime} \models \operatorname{Pre}(b)$ and $M, u \models \hat{B}_{j} \operatorname{Pre}(b)$. So $M, u \models[a] \hat{B}_{j}\left\langle b^{-}\right\rangle \top$ by Definition 4.$](4)$. But $h a \in \mathcal{H}$. So $\mathcal{R}_{a}(u) \neq \emptyset$. Therefore $M, u=\langle a\rangle \hat{B}_{j}\left\langle b^{-}\right\rangle \top$, i.e. $\mathcal{R}_{a} \circ R_{j} \circ \mathcal{R}_{b}^{-1} \neq$ $\emptyset$.

So by the no-learning constraint $R_{j} \circ \mathcal{R}_{b}(u) \subseteq \mathcal{R}_{a} \circ R_{j}(u)(3)$. But $h^{\prime} b \in \mathcal{H}$. So $\mathcal{R}_{b}\left(u^{\prime}\right) \neq \emptyset$. Therefore there is $v \in \mathcal{R}_{b}\left(u^{\prime}\right)$, so $v \in R_{j} \circ \mathcal{R}_{b}(u)$. So $v \in \mathcal{R}_{a} \circ R_{j}(u)$ by (3). Finally $u^{\prime} \in \mathcal{R}_{a} \circ R_{j} \circ \mathcal{R}_{b}^{-1}(u)$.

Bisimulation invariance Let $h, h^{\prime} \in \mathcal{H}$ which are epistemically bisimilar such that $h a \in \mathcal{H}$. Then we have $h=w a_{1} \ldots a_{n}$ and $h^{\prime}=w a_{1}^{\prime} \ldots a_{n}^{\prime}$. Let $u=\mathcal{R}_{a_{1}} \circ \ldots \circ \mathcal{R}_{a_{n}}(w) \in W$ and $u^{\prime}=\mathcal{R}_{a_{1}^{\prime}} \circ \ldots \circ \mathcal{R}_{a_{n}^{\prime}} \in W$. Then $\mathcal{R}_{a}(u) \neq \emptyset$, so $M, u \models \operatorname{Pre}(a)$. Therefore $M, u^{\prime}=\operatorname{Pre}(a)$ because $u$ and $u^{\prime}$ are epistemically bisimilar. So $M, u^{\prime}=\langle a\rangle \top$ by Definition 4.] (3), i.e. $\mathcal{R}_{a}\left(u^{\prime}\right) \neq \emptyset$. So $h^{\prime} a \in \mathcal{H}$.

QED

Note that the converse of Proposition 5.9 does not hold in general for the same reason that the right to left direction of Theorem 5.4 does not hold in general if we adopt the standard BMS framework.

\subsection{Other related work}

Still in the ETL paradigm the authors in [26] show how to translate a BMS formula satisfied in a static model into an ETL formula satisfied in an interpreted system. So their approach is less general than ours because it only deals with the model checking problem. Starting from the BMS formalism, Yap [28] and Sack [17, 16] introduce a 'yesterday' temporal modal operator to the BMS language expressing what was true before the last event; Sack gets a complete characterization. To prove completeness Sack [16] also introduces a separate component expressing that an event just occurred but this is not a converse modal operator like ours. However he does introduce a converse modal operator for public announcement logic but does not provide a completeness proof for it [17].

Another approach embedding the BMS formalism to a formalism that also deals with events and beliefs on the same formal level is proposed by van Eijck et col. in [27, 25]. They map the BMS formalism to (epistemic) propositional dynamic logic (refining a similar result for automata propositional dynamic logic [22]). However they do not resort to converse events and translate directly event models into a transformation on PDL programs. 
In a previous publication of ours [2], the constraint of no-forgetting and condition (3) on $\Gamma(A)$ of Definition 4.T on EDL-models of Definition 2.2 were replaced by the following ones

nf' if $v\left(\mathcal{R}_{a} \circ R_{j} \circ \mathcal{R}_{b}^{-1}\right) v^{\prime}$ then $v R_{j} v^{\prime} ;$

$(3)^{\prime} \vdash_{\mathrm{BMS}}[a] B_{j} \varphi \leftrightarrow\left(\operatorname{Pre}(a) \rightarrow B_{j}\left[a_{1}\right] \varphi \wedge \ldots \wedge B_{j}\left[a_{n}\right] \varphi\right)$

where $a_{1}, \ldots, a_{n}$ is the list of all $b$ such that $b \in R_{j}(a)$.

Neither do EDL models satisfy nf', nor the other way round. Hence the version of EDL in [2] cannot be compared with our present version. If we moreover assume that event models are serial then we obtain the same results as here. Here we do not need this last assumption and our condition (3) describes more accurately than (3)' the structure of event models. Our constraint nf is also a better generalization of the principle of perfect-recall than $\mathrm{nf}^{\prime}$.

\section{Conclusion}

We have presented an epistemic dynamic logic EDL whose semantics differs from the BMS semantics. We have shown that BMS can be embedded into EDL. This result allows to conclude that EDL is an interesting alternative to Baltag et al.'s logic, that allows to talk about agents' perception of events just in the same way as BMS does. However, EDL is more expressive than BMS because it allows to talk about past events. Another of its advantages is that EDL allows for incomplete beliefs about the event taking place and can still draw inferences from this incomplete description of the event, while in BMS the event model has to specify everything. So in a sense EDL seems more versatile than BMS to describe events.

On the other hand, the power of event models (actually called action models in BMS) is not completely exploited in the BMS approach. Indeed, the philosophy of the BMS approach is to represent events in the same way as situations are represented in epistemic logic by means of static models. But unlike a static model, an event model does not have a genuine valuation to describe possible events. An obvious extension of the BMS formalism would be to add a valuation to event models in order to describe possible events more precisely. Then we could define epistemic languages for event models completely identical to the various epistemic languages we already defined for static models, except that the propositional letters of these languages would describe possible events instead of possible worlds. This would allow to express things about events that are currently taking place, and not only to express things before or after the occurrence of events as in EDL. This would also allow to update/revise events by other events which is a phenomenon that often occurs in everyday life.It is not possible to model such phenomena in EDL because the accessibility relations for events are set once and for all. This idea is explored in [T]. 


\section{References}

[1] Guillaume Aucher. Bms revisited. In Proceedings of Theoretical Aspects of Rationality and Knowledge (TARK 2009), 2009. To appear.

[2] Guillaume Aucher and Andreas Herzig. From DEL to EDL: exploring the power of converse events. In European Conference on Symbolic and Quantitative Approaches to Reasoning with Uncertainty (ECSQARU), Hammamet, volume 4724 of LNCS, pages 199-209. Springer, 2007.

[3] Alexandru Baltag and Larry Moss. Logic for epistemic programs. Synthese, 139(2):165-224, 2004.

[4] Alexandru Baltag, Larry Moss, and Slawomir Solecki. The logic of common knowledge, public announcement, and private suspicions. In I. Gilboa, editor, Proceedings of the 7th conference on theoretical aspects of rationality and knowledge (TARK98), pages 43-56, 1998.

[5] Alexandru Baltag and Lawrence S. Moss. Logics for epistemic programs. Synthese, 139(2):165-224, 2004.

[6] Alexandru Baltag, Lawrence S. Moss, and Slawomir Solecki. The logic of public announcements, common knowledge, and private suspicions. In Proc. TARK'98, pages 43-56. Morgan Kaufmann, 1998.

[7] Tiago de Lima. Optimal Methods for Reasoning About Actions and Plans in Multi-agent Systems. PhD thesis, Université Paul Sabatier, Toulouse, 2007.

[8] Ronald Fagin, Joseph Halpern, Yoram Moses, and Moshe Vardi. Reasoning about knowledge. MIT Press, 1995.

[9] Jelle Gerbrandy. Bisimulations on Planet Kripke. PhD thesis, University of Amsterdam, 1999.

[10] David Harel, Dexter Kozen, and Jerzy Tiuryn. Dynamic Logic. MIT Press, 2000.

[11] Andreas Herzig, Jérôme Lang, Dominique Longin, and Thomas Polacsek. A logic for planning under partial observability. In $A A A I / I A A I$, pages 768-773, 2000.

[12] Hirofumi Katsuno and Alberto O. Mendelzon. On the difference between updating a knowledge base and revising it. In Peter Gärdenfors, editor, Belief revision, pages 183-203. Cambridge University Press, 1992. (preliminary version in Allen, J.A., Fikes, R., and Sandewall, E., eds., Principles of Knowledge Representation and Reasoning: Proc. 2nd Int. Conf., pages 387-394. Morgan Kaufmann Publishers, 1991).

[13] Eric Pacuit. Some comments on history based structures. Journal of Applied Logic, 5(4):613-624, 2007.

[14] Rohit Parikh and Ramaswamy Ramanujam. A knowledge based semantics of messages. Journal of Logic, Language and Information, 12(4):453-467, 2003.

[15] J. A. Plaza. Logics of public communcations. In M. L. Emrich, M. Z. Pfeifer, M. Hadzikadic, and Z. W. Ras, editors, Proc. 4th Int. Symposium on Methodologies for Intelligent Systems, pages 201-216, 1989.

[16] Joshua Sack. Logic for update products and steps into the past. Submitted to Annals of Pure and Applied Logic, 2007.

[17] Joshua Sack. Temporal languages for epistemic programs. Journal of Logic, Language and Information, 17(2):183-216, 2008. 
[18] Henrik Sahlqvist. Completeness and correspondence in the first and second order semantics for modal logics. In Stig Kanger, editor, Proceedings of the 3rd Scandinavian Logic Symposium 1973, number 82 in Studies in Logic. North Holland, 1975.

[19] Krister Segerberg. Belief revision from the point of view of doxastic logic. Bulletin of the IGPL, 3:534-553, 1995.

[20] Krister Segerberg. Two traditions in the logic of belief: bringing them together. In Hans Jürgen Ohlbach and Uwe Reyle, editors, Logic, Language and Reasoning: essays in honour of Dov Gabbay, volume 5 of Trends in Logic, pages 135-147. Kluwer Academic Publishers, Dordrecht, 1999.

[21] Johan van Benthem, Jelle Gerbrandy, and Eric Pacuit. Merging frameworks for interaction: DEL and ETL. In Dov Samet, editor, Theoretical Aspect of Rationality and Knowledge (TARK XI), pages 72-82, Brussels, June 2007.

[22] Johan van Benthem and Barteld Kooi. Reduction axioms for epistemic actions. In R. Schmidt, I. Pratt-Hartmann, M. Reynolds, and H. Wansing, editors, AiML-2004: Advances in Modal Logic, number UMCS-04-9-1 in Technical Report Series, pages 197-211, University of Manchester, 2004.

[23] Johan van Benthem and Fenrong Liu. Diversity of agents in games. Philosophia Scientiae, 8(2), 2004.

[24] Johan van Benthem and Eric Pacuit. The tree of knowledge in action: Towards a common perspective. In Advances in Modal Logic, pages 87-106, 2006.

[25] Johan van Benthem, Jan van Eijck, and Barteld Kooi. Logics of communication and change. Information and Computation, 204(11):1620-1662, 2006.

[26] Hans van Ditmarsch, Ji Ruan, and Wiebe van der Hoek. Model checking dynamic epistemics in branching time. In Formal Approaches to Multi-agent Systems 2007 (FAMAS 2007), Durham UK, 2007.

[27] Jan van Eijck. Reducing dynamic epistemic logic to pdl by program transformation. Technical Report SEN-E0423, CWI, 2004.

[28] Audrey Yap. Product update and looking backward. prepublications PP-2006-39, ILLC, 2006. 